\title{
Possible Geometries for Afterglow of GRB990705
}

\author{
X. Y. Wang, Z. G. Dai, and T. Lu \\ Department of Astronomy,Nanjing University, Nanjing 210093, \\ P.R.China
}

\begin{abstract}
We investigate whether the circum-burst environment constrained by the absorption feature of GRB990705 could be consistent with the observed $H$-band afterglow. Two possible geometries of the afterglowemitting regions are suggested: 1) afterglow emission produced by the impact of the fireball on the surrounding torus; 2)afterglow emission produced in the dense circum-burst medium inside the torus. In case 1), the faster decay at later time is attributed to the disappearance of the shock due to the counter-pressure in the hot torus illuminated by the burst and afterglow photons. For case 2), the circum-burst medium density is found to be very high $\left(n \gtrsim 10^{4}-10^{5} \mathrm{~cm}^{-3}\right)$.
\end{abstract}

\section{Afterglow models for GRB990705}

The absorption feature of GRB990705 was originally explained by Amati et al. (2000) as being due to an edge produced by neutral iron redshifted to $3.8 \pm$ $0.3 \mathrm{keV}$, which was, however, questioned by Lazzati et al. (2001) as it requires a vast amount of iron in the close vicinity of the burster. Lazzati et al. (2001) further suggested an alternative scenario in which the feature is produced by resonant scattering from hydrogen-like iron broadened by a range of outflow velocities. In this scenario, the radius of the $\mathrm{SN}$ shell of the supranova-like models (Vietri \& Stella 1998; Wang et al. 2000) is fixed by the requirement that the heating timescale of the electrons in the absorbing matter is $\sim 10 \mathrm{~s}$, i.e. $R_{s} \sim(2-3) \times 10^{16} \mathrm{~cm}$.

According to $R_{d} \gg R_{s}$ or $R_{d} \ll R_{s}$ ( $R_{d}$ is the deceleration radius of the GRB shell), there are two possible geometries of the afterglow-emitting regions: one is in the torus on which the fireball impacts (case I) and the other is in the circum-burst medium inside the torus (case II).

case I (jet-torus interaction model): The impact of the fireball on the torus will generate a forward shock propagating into the torus, and a reverse one moving into the fireball shell (Vietri et al. 1999). We show that, for GRB990705 which has a much larger shock energy $E$, the forward shock could last few days ( especially for the lower estimated value for the torus temperature by Paerels et al. (2000) , see Eq. (7)), giving rise to an early power-law fading afterglow as seen in GRB990705. The disappearance of this forward shock, caused by the strong counter-pressure in the pre-shock torus, may just account for the observed faster decline at late time. 


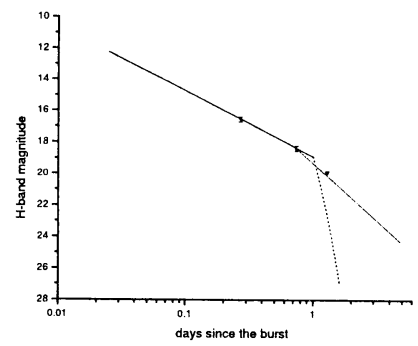

Figure 1. The solid line represents the power-law decay of the afterglow as the forward shock slows down in the tours and the thick dotted line represents the late exponential decay of this shock due to the effect of the counter-pressure in the hot torus. The thin dotted line represents the late power-law decay $F_{\nu} \propto t^{-p}$ due to jet behavior.

The $H$-band decay index of GRB990705 before 1 day can be reproduced if $p \simeq 2.8$ and $\nu_{H}>\nu_{c}>\nu_{m}$. In Fig.1, we give an analytic fitting to the $H$-band afterglow. For a SN shell of $10 M_{\odot}$ mass with a typical width $\Delta R=10^{15} \mathrm{~cm}$ at this radius, its particle density is $n_{s}=10^{9} \mathrm{~cm}^{-3}$. We also chose the shock energy to be $E=5 \times 10^{53} \mathrm{erg}$. We find that the following values for these parameters are consistent with the observations: $\epsilon_{e}=0.5, \epsilon_{B}=10^{-5}$ and $\theta_{j}=0.2$. The solid line in Fig. 1 represents the power-law decay of the afterglow as the forward shock slows down in the tours and the thick dotted line represents the later exponential decay $\left(F_{\nu} \propto \exp (-\mathrm{t})\right)$ of this shock due to the counter-pressure in the hot torus.

Case II (jet in a dense circum-burst medium): The steepness of the light curve decay could also be produced by a usual beamed outflow. Assuming that a break due to jet sideways spreading occurs in the $H$-band light curve of GRB990705 about one day after the burst, the early time slope $\alpha \simeq 1.68$ and the later one $\alpha^{\prime}>2.6$ would be consistent with $p \sim 2.9$. For a uniform circum-burst medium, we have $R_{b}=7 \times 10^{17} \mathrm{~cm} E_{0,51}^{1 / 3} n_{0}^{-1 / 3}$ where $E_{0}$ is the actual energy of the jet, $E_{0}=E_{\text {iso }} \theta_{j}^{2} / 2$. Requiring $R_{b}<R_{s} \sim 3 \times 10^{16} \mathrm{~cm}$, means that the circum-burst medium $n \gtrsim 10^{4}-10^{5} \mathrm{~cm}^{-3}$, even if the actual energy of the burst is only $E_{0} \sim$ a few $\times 10^{51} \mathrm{erg}$ as founded by Frail et al. (2001).

\section{References}

Amati, L., Frontera, F., Vietri, M. et al. 2000, Science, 290, 953

Frail, D. A., Kulkarni, S. R., Sari, R. et al. 2001, ApJ, 562, L55

Lazzati, D., Ghisellini, G., Amati, L. et al. 2001, ApJ, 556, 471

Paerels, F., Kuulkers, E., Heise, J., \& Liedahl, D. A. 2000, ApJ, 535, L25

Vietri, M. \& Stella, L. 1998, ApJ, 507, L45

Vietri, M., Perola, G. C., Piro, L., \& Stella, L. 1999, MNRAS, 308, L29.

Wang, X. Y. Dai, Z. G., Lu, T. et al. 2000, A\&A, 357, 543 